\title{
Análise de prontuários de indivíduos submetidos ao tratamento para hanseníase em
}

\section{uma unidade básica de saúde}

\author{
Analysis of research records for leprosy treatment in a basic health unit \\ Análisis de historias clínicas de investigación para el tratamiento de la lepra en una unidad básica de \\ salud
}

Recebido: 22/09/2021 | Revisado: 28/09/2021 | Aceito: 20/09/2021 | Publicado: 22/09/2021

\author{
Alessandra Camillo da Silveira Castello Branco \\ ORCID: https://orcid.org/0000-0001-8877-0461 \\ Centro Universitário Santo Agostinho, Brasil \\ E-mail: alessandracscb@gmail.com \\ Dione da Conceição Nunes \\ ORCID: https://orcid.org/0000-0001-9234-0800 \\ Centro Universitário Santo Agostinho, Brasil \\ E-mail: dionenunes29@ outlook.com \\ Francisca Ranielle Lima Leite \\ ORCID: https://orcid.org/0000-0002-8049-4623 \\ Centro Universitário Santo Agostinho, Brasil \\ E-mail: ranielleranielleleite@gmail.com
}

\begin{abstract}
Resumo
A hanseníase é uma doença crônica, causada pelo Mycobacterium leprae, que acomete a pele causando lesões. No entanto, o agente infeccioso também possui predileção pelos nervos periféricos, ocasionando o comprometimento das funções motoras quando não tratada. O diagnóstico precoce é fundamental para controle e eliminação da doença. Desta forma para uma terapia medicamentosa adequada é necessário a orientação. A presente pesquisa teve como objetivo a análise do tratamento farmacológico e não farmacológico de indivíduos com hanseníase cadastrados em uma unidade básica de saúde (UBS) da Zona Sul da cidade de Teresina, no Piauí em um período de 10 anos (2011-2021) mediante avaliação de prontuários e, consequentemente, avaliação da sua qualidade de vida e forma de adesão a terapia. O estudo tratou-se de uma pesquisa transversal documental retrospectiva de caráter descritivo, com abordagem qualiquantitativa, nas quais os dados foram coletados por meio da avaliação dos prontuários de pacientes tratados no intervalo dos anos de 2011 à 2021. Mediante os resultados obtidos, evidenciou-se a ausência do serviço de atenção farmacêutica e a sua direta contribuição para a ineficácia do tratamento farmacológico e não farmacológico para hanseníase, somado ao desconhecimento dos pacientes acerca do real conhecimento sobre a patologia - o que corroborou para a dificuldade de adesão ao tratamento, somado aos efeitos adversos passíveis de prevenção causados pela poliquimioterapia, evidenciando assim mais uma vez a necessidade do profissional farmacêutico como o responsável em conjunto pela terapia medicamentosa do paciente em tratamento para hanseníase.
\end{abstract}

Palavras-chave: Hanseníase; Mycobacterium leprae; Problemas relacionados à medicamentos; Seguimento Farmacoterapêutico.

\begin{abstract}
Leprosy is a chronic disease, caused by Mycobacterium leprae, which affects the skin causing lesions. However, the infectious agent also has a predilection for peripheral nerves, causing impairment of motor functions when left untreated. Early diagnosis is essential for controlling and eliminating the disease. Therefore, for an adequate drug therapy, guidance is necessary. This research aimed to analyze the pharmacological and non-pharmacological treatment of individuals with leprosy registered in a basic health unit (UBS) in the South Zone of the city of Teresina, Piauí, over a period of 10 years (2011-2021) by evaluation of medical records and, consequently, evaluation of their quality of life and form of adherence to therapy. The study was a retrospective cross-sectional descriptive documentary research, with a qualiquantitative approach, in which data were collected through the assessment of the medical records of patients treated in the period from 2011 to 2021. if the absence of the pharmaceutical care service and its direct contribution to the ineffectiveness of pharmacological and non-pharmacological treatment for leprosy, added to the patients' lack of real knowledge about the pathology - which corroborated the difficulty of adherence to treatment, added the preventable adverse effects caused by multidrug therapy, thus demonstrating once again the need for the pharmacist to be jointly responsible for the drug therapy of the patient being treated for leprosy.
\end{abstract}

Keywords: Leprosy; Mycobacterium leprae; Problems related to medications; Pharmacotherapeutic follow-up. 


\section{Resumen}

La lepra es una enfermedad crónica, causada por Mycobacterium leprae, que afecta la piel provocando lesiones. Sin embargo, el agente infeccioso también tiene predilección por los nervios periféricos, lo que causa deterioro de las funciones motoras cuando no se trata. El diagnóstico precoz es fundamental para controlar y eliminar la enfermedad. Por lo tanto, para una terapia farmacológica adecuada, se necesita orientación. Esta investigación tuvo como objetivo analizar el tratamiento farmacológico y no farmacológico de personas con lepra registradas en una unidad básica de salud (UBS) en la Zona Sur de la ciudad de Teresina, Piaú, durante un período de 10 años (2011-2021) mediante evaluación. de historias clínicas y, en consecuencia, evaluación de su calidad de vida y forma de adherencia a la terapia. El estudio fue una investigación documental descriptiva, transversal, retrospectiva, con enfoque cualitativo, en la que se recogieron datos a través de la valoración de las historias clínicas de los pacientes atendidos en el período 2011 a 2021. si la ausencia del servicio de atención farmacéutica y su Contribución directa a la ineficacia del tratamiento farmacológico y no farmacológico de la lepra, sumado al desconocimiento real de la patología por parte de los pacientes - lo que corroboró la dificultad de adherencia al tratamiento, sumado los efectos adversos prevenibles provocados por la multidroga, demostrando así una vez de nuevo la necesidad de que el farmacéutico sea corresponsable de la farmacoterapia del paciente en tratamiento de lepra.

Palabras clave: Lepra; Mycobacterium leprae; Problemas relacionados con los medicamentos; Seguimiento farmacoterapéutico.

\section{Introdução}

A hanseníase é uma doença crônica, granulomatosa, causada pelo Mycobacterium leprae que afeta pele, sistema nervoso periférico e, ocasionalmente, outros órgãos e sistemas. Vários estudos têm demonstrado que, diante da contaminação, a maioria dos indivíduos oferece resistência ao M. leprae, não desenvolvendo a doença - situação que pode ser alterada em função da relação entre agente, meio ambiente e hospedeiro (Orsini, 2007).

Ocasionada pelo bacilo de Hansen - parasita intracelular obrigatório, fracamente gram-positivo, a sua multiplicação ocorre de forma progressiva e lenta, podendo durar, em média, 11 a 16 dias, com elevado poder infectante e baixa patogenicidade - isto é, infecta várias pessoas, no entanto poucas apresentam os sintomas. O homem é reconhecido como o único hospedeiro para o parasita (Brasil, 2002).

Conforme Brasil (2001), se o M. leprae acometesse somente a pele, a hanseníase não teria a importância que tem à nível de saúde pública. Somando-se as lesões da pele, o agente infectante apresenta predileção pelos nervos periféricos - sistema nervoso periférico (SNP) - e se não tratada em tempo a doença pode proporcionar uma redução dos sentidos comprometendo a atividade funcional, isolando o indivíduo doente, não permitindo a sua interação com o meio em que vive.

Pelo fato do acometimento do SNP surge a perda da sensibilidade, as atrofias, paresias - disfunções marcadas pela interrupção dos movimentos de um ou mais membros segundo o grau do comprometimento - e paralisias musculares, que se não diagnosticadas e tratadas adequadamente, podem evoluir para incapacidades físicas permanentes. Além das deformidades físicas e neurológicas, Eidt (2000) chama a atenção para as repercussões psicológicas ocasionadas pelas sequelas físicas da doença como fatores contribuintes para a diminuição da autoestima e para auto segregação do hanseniano.

O diagnóstico precoce é crucial para o controle da doença e eliminação do estigma. Contudo, em muitos casos, a pessoa passa despercebida com a doença. Segundo Brasil (2008), muitos pacientes passam anos com sinais e sintomas, consultam médicos que por vezes são dermatologistas e ainda assim não são diagnosticados. Outros pacientes recebem o diagnóstico já com sequelas permanentes e perdem um tempo precioso de tratamento e prevenção de incapacidades.

Durante muitos anos, o único tratamento contra a hanseníase foi o óleo de "chalmoogra", cuja origem se perde nos domínios da lenda. Sua ação, contudo, era muito discutível, pois os casos em que aparentava ter alguma atividade eram casos que costumam regredir espontaneamente (Opromolla, 1997). No início da década de quarenta, nos Estados Unidos, Faget experimentou uma sulfona - o "Promin" - no tratamento da hanseníase, com bons resultados. Outras sulfonas, também ativas, apareceram e todas derivadas de um composto "mãe", a diamino-difenilsulfona. Contudo, verificou-se, mais tarde, que quem 
atuava mesmo contra a doença era esse composto "mãe", porque todas as drogas que o compunham acabavam depurando-se no organismo (Lima, 1953).

Depois da sulfona, outros medicamentos foram aparecendo com atividade anti-hansênica - tanto quimioterápicos como antibióticos - mas dentre todos eles, os que são considerados hoje como de primeira linha são a sulfona, a clofazimina e a rifampicina. O tratamento já é iniciado na primeira consulta através da associação de medicamentos (poliquimioterapia - PQT) segundo a definição do diagnóstico, se não houver contraindicações formais (alergia à sulfa ou à rifampicina).

Os medicamentos mais utilizados na Hanseníase - além da PQT - são os fármacos para controle das patologias envolvidas, normalmente corticosteroides, analgésicos, dentre outros anti-inflamatórios. É importante a observação da ocorrência de interações medicamentosas entre os mesmos, pois podem causar efeitos adversos em tais pacientes, abandono ao tratamento farmacológico e agravamento em seu estado de saúde (Opromolla, 1997).

Deste modo, o farmacêutico é essencial para orientação dos pacientes, pois os indivíduos que apresentam hanseníase geralmente utilizam medicamentos associados visando evitar complicações oriundas da terapia anti-hansênica. Desta forma, torna-se importante a introdução de um acompanhamento farmacoterapêutico para tais pacientes objetivando uma adequada e eficaz terapêutica, além de minimizar os efeitos adversos ocasionados pela medicação e posterior sucesso na farmacoterapia (Gomes, 2015).

A atenção farmacêutica em pacientes com hanseníase é indispensável, para que se consiga alcançar um elevado sucesso com os fármacos usados, garantindo ao paciente que os medicamentos prescritos sejam seguros e eficazes, resultando no efeito terapêutico desejado e minimizando efeitos adversos. Assim, cabe ao farmacêutico toda a orientação sobre a forma de uso e a administração desses medicamentos, sempre orientando os pacientes de uma maneira clara, podendo ser em seu domicílio para aqueles indivíduos que se encontram frágil, ou então nas unidades básicas de saúde (UBS’s). A atenção farmacêutica deve ser prestada não só em relação ao modo de usar o medicamento, mas também fornecendo toda a orientação quanto à promoção e prevenção da saúde (Azevedo, 2015).

Entendendo o papel indispensável da adesão ao tratamento como meio de alcançar os objetivos terapêuticos, observase que o farmacêutico deve promover o uso racional de medicamentos por meio da atenção farmacêutica em que o paciente é acompanhado individualmente e são verificadas as questões que podem interferir na adesão ao tratamento como: orientações sobre possíveis efeitos adversos, buscando possíveis interações medicamentosas ou com alimentos e verificando os possíveis resultados negativos relacionados aos medicamentos, sendo que todo ao acompanhamento deve ser planejado e documentado (Silva, 2016).

Mediante tais fatos abordados, este estudo tem como objetivo analisar o tratamento farmacológico e não farmacológico de indivíduos com hanseníase cadastrados em uma UBS da Zona Sul de Teresina-Piauí em um período de 10 anos (2011-2021) mediante avaliação de prontuários e, consequentemente, examinando o perfil sócio demográfico de tais pacientes, sua adesão farmacológica à poliquimioterapia e qualidade de vida.

\section{Metodologia}

A pesquisa trata-se de um estudo transversal documental retrospectivo de caráter descritivo, com abordagem qualiquantitativa, mediante ao uso de um questionário.

A pesquisa descritiva observa, registra, analisa e correlacionam os fenômenos e fatos variáveis apresentados, sem manipulá-los, sendo realizada através de técnicas padronizadas de coletas de dados como observação sistemática e questionários (Gil, 2002; Cervo; Bervian, 2002). A abordagem qualitativa utiliza-se no estudo das representações, das crenças, dos olhares, das relações e concepção do sujeito investigador (Minayo, 1993). Já a quantitativa foi combinada com os dados qualitativos 
originados de observações, entrevistas - onde buscou abranger as características e fatores que constituem um determinado fenômeno social (Creswell, 2007).

A coleta de dados aconteceu inicialmente por meio da avaliação de dados descritos no prontuário de cada paciente, contendo dados pessoais (nome, idade), histórico de tratamento, com os dados das prescrições. Em seguida avaliou-se os parâmetros que englobam o tratamento terapêutico, as reações adversas descritas no prontuário, período de tratamento e fármacos usados para o tratamento de outras patologias.

Os dados foram coletados no período de abril a junho de 2021 por meio da avaliação dos prontuários eletrônicos e dos prontuários físicos presentes na instituição correspondente aos intervalos de anos de 2011 a 2021. Para a realização desta pesquisa, foram utilizados formulários para a coleta de dados com questões que foram pré-elaboradas mediante fichamento prévio para a análise das questões de acordo com o prontuário utilizado na UBS.

As variáveis coletadas foram sócio demográficas - tais como sexo, idade, estado civil e escolaridade -, clínicas, abordando as variáveis do tratamento medicamentoso - tais como diagnóstico principal (CID contemplada), data do início e término do tratamento, como foi detectado a doença, qual a forma clínica da hanseníase, qual esquema terapêutico inicial, reação adversa apresentada pelo paciente aos medicamentos rifampicina/dapsona/clofazimina, outras doenças diagnosticadas, outros medicamentos utilizados somados à PQT relatados, horário de uso da PQT, se o paciente se esquece de tomar a medicação, se utiliza bebidas alcoólicas, se já foi encaminhado ao Centro de Referência para Hanseníase - e as abordando as variáveis do tratamento não medicamentoso - como prática de exercício físico, realização de sessões de fisioterapia para auxílio do tratamento da hanseníase e histórico familiar (se algum parente e/ou pessoa do convívio diário já veio à apresentar tal patologia).

Em conjunto à tais variáveis, considerou-se e avaliou-se as informações contidas nos prontuários inerente ao relatado pelo profissional de enfermagem responsável pelo preenchimento e acompanhamento do paciente durante o diagnóstico e todo tratamento, até sua alta por critérios de cura.

Este trabalho foi aprovado pela Comissão de Ética em Pesquisa da Fundação Municipal de Saúde do estado do Piauí (FMS-PI) sob MEMO CEP/FMS N 16/2021 e MEMO CEP/FMS N¹7/2021 e pelo Comitê de Ética e Pesquisa do Centro Universitário Santo Agostinho (UNIFSA) sob parecer número 4.732.006.

Os dados coletados dos prontuários durante a pesquisa foram tabulados e analisados através do programa estatístico PRISMA 5.0, e expressos em forma de gráficos e tabelas através de planilhas no Microsoft Office Excel. Ressalta-se que para calcular o tamanho da amostra utilizou-se o software Raosoft Sample Size ${ }^{\circledR}$ considerando-se que a população de referência é igual 50, com o nível de confiança é de 95\% e nível de erro de 5\%, portanto o tamanho mínimo recomendado da amostra foi de 45.

Visando agregar e comparar os dados coletados da UBS com uma visão epidemiológica de todo o escopo da doença no município de Teresina-PI no mesmo intervalo de anos, realizou-se uma posterior coleta de dados nos meses de abril a maio de 2021 no Sistema de Informação Hospitalar (SIH) do Departamento de Informática do Sistema Único de Saúde (DATASUS). Foi utilizado - a partir da função "Informações Epidemiológicas e Morbidade" - o subgrupo Casos de Hanseníase desde 2001 (SINAN). Na abrangência geográfica, para a coleta de dados referentes ao município de Teresina no período de 2011 a 2020, foi selecionado o estado do Piauí. Ressalta-se que se utilizou tal período de anos decorrente da ausência de informações no sistema no ano de $2021 \mathrm{em}$ todas as abas de pesquisa fornecidos.

A população deste estudo foi composta por todos os pacientes diagnosticados com o código da Décima Revisão da Classificação Internacional de Doenças e Causas de Mortalidade (CID-10) para Hanseníase (CID 10 - A30), Hanseníase indeterminada (CID 10 - A30.0), Hanseníase tuberculóide (CID 10 - A30.1), Hanseníase tuberculóide bonderline (CID 10 A30.2), Hanseníase dimorfa (CID 10 - A30.3), Hanseníase lepromatosa bonderline (CID 10 - A30.4) e Hanseníase lepromatosa 
(CID 10 - A30.5), os quais o município de Teresina foi o de notificação e residência no período delimitado pelo estudo (2011 a 2020) e que foram contabilizados no formulário eletrônico do DATASUS.

Foram coletadas as seguintes variáveis: Casos notificados - residentes em Teresina e segundo o sexo -, forma clínica notificada, baciloscopia e esquemas terapêuticos. Neste estudo, as faixas etárias foram categorizadas de 20 a 75 anos segundo sexo assim como raça mediante ano diagnóstico para melhor análise de dados.

Mediante os dados coletados do SIH, os mesmos foram transportados para uma planilha do programa Microsoft Office Excel, em que as linhas representavam o período delimitado na pesquisa (2011 a 2020) e as colunas representam as variáveis a serem analisadas. As variáveis, todas do tipo quantitativas, foram tabuladas e apresentadas como dados absolutos e, posteriormente, quando necessário, calculou-se as porcentagens utilizando o mesmo programa.

Como complemento de tal estudo, utilizou-se o software Medscape® que auxiliou na detecção das possíveis interações medicamentosas observadas nos prontuários de tratamento para hanseníase - onde avaliou-se as possíveis interações entre os fármacos pertencentes tanto à poliquimioterapia como os de uso contínuo pelo paciente para o tratamento de outras comorbidades - que foram classificadas posteriormente em três classes de acordo com a significância da interação medicamentosa e posteriores subclasses.

\section{Resultados}

No Gráfico 1 têm-se os dados referentes ao total de número de casos de hanseníase notificados residentes em TeresinaPI no período de 2011 a 2020, que totalizaram 4.147 casos novos, sendo que, destes, 1.955 ocorreram com mulheres e 2.192 em homens - onde o sexo masculino representou $52,85 \%$ do número total de casos (Gráfico 2). Embora seja uma porcentagem pequena em comparação ao sexo feminino, observa-se um certo crescimento anual contínuo em comparação ao sexo feminino e, decorrente do atual contexto da pandemia da COVID-19, notou-se um decréscimo na quantidade de casos notificados - mas ainda sim com predominância de notificação pelo sexo masculino.

\section{Gráfico 1: Casos notificados residentes em Teresina-Pi no intervalo de anos de 2011 à 2020 \\ Casos notificados em Teresina}

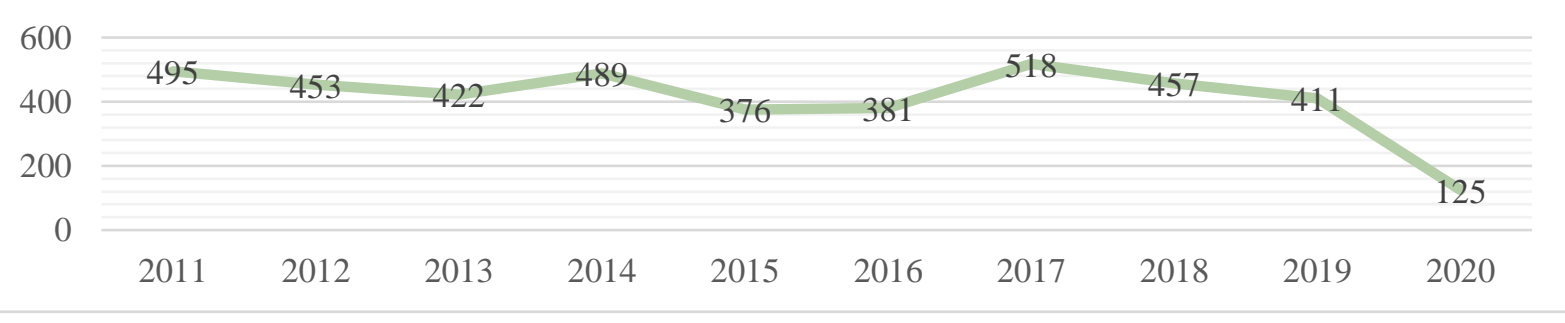

Fonte: Ministério da Saúde/SVS - Sistema de Informações de Agravos de Notificação - Sinan Net (2021). 


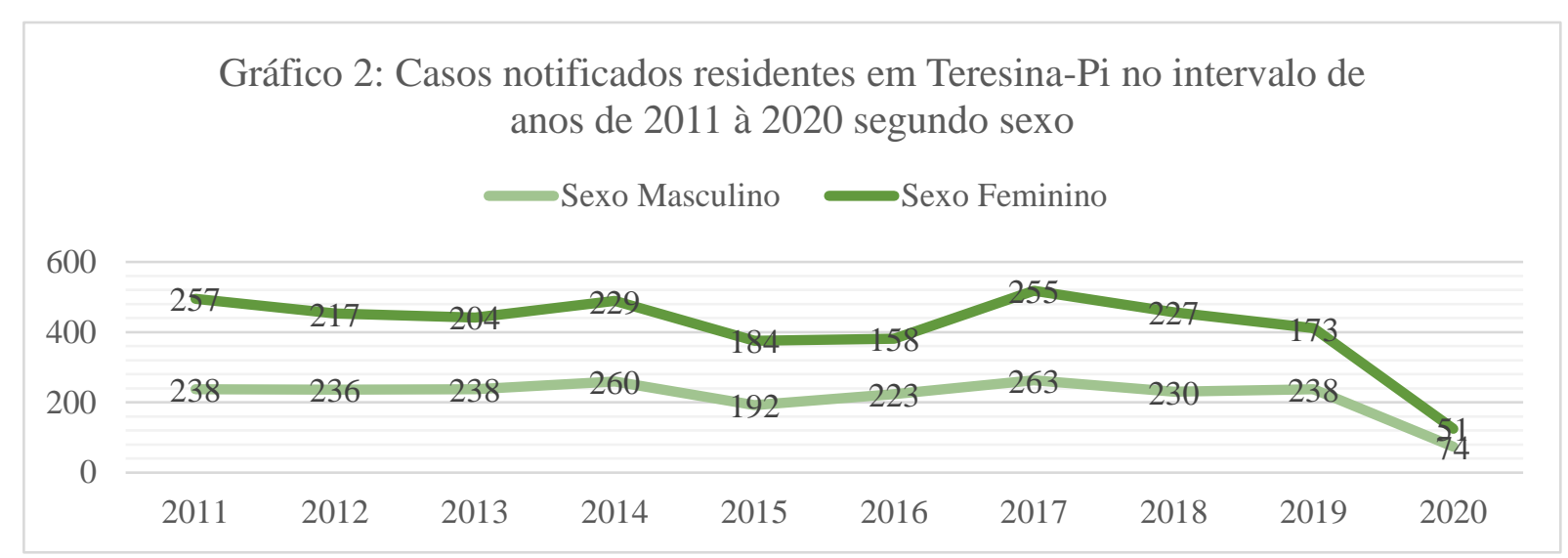

Fonte: Ministério da Saúde/SVS - Sistema de Informações de Agravos de Notificação - Sinan Net (2021).

Em comparação aos dados coletados na Unidade Básica de Saúde São Camilo, obteve-se um total de 26 homens e 20 mulheres, totalizando 46 pacientes na amostra - onde utilizou-se o software Raosoft Sample Size ${ }^{\circledR}$ considerando-se que a população de referência de 50 pacientes, com o nível de confiança é de $95 \%$ e nível de erro de 5\%, o tamanho mínimo recomendado da amostra foi de 45 e obteve-se 46 prontuários de pacientes.

Em relação à faixa etária de diagnóstico entre 2011 e 2021 observado nos prontuários avaliados, o maior número de casos englobou a faixa etária entre 60 a 69 anos - o que reforça as projeções de que se tratando da hanseníase no idoso ( $\geq 60$ anos de idade), há registros de aumento gradativo do número de casos novos, uma vez que, em 2017 foram diagnosticados 6.598 casos novos e, em 2018, 6.739 casos (DATASUS, 2019). Como complemento, observou-se que 28,26\% dos pacientes avaliados apresentou algum dos níveis de escolaridade completo - primário, fundamental, médio e superior -, enquanto $58,70 \%$ relataram terem cursado, mas não completado tais etapas de aprendizagem, 2,18\% relataram ser analfabetos e 10,86\% não informou o grau de escolaridade.

Quanto à forma clínica de acordo com os dados coletados do DATASUS (Gráfico 3), observou-se a prevalência da forma dimorfa - com predomínio no sexo masculino -, seguido da forma tuberculóide - predominante no sexo feminino -, indeterminada e virchowiana. Ressalta-se que no ano de 2015 houve uma notável queda em relação à notificação de tais formas clínicas no sexo masculino - com ênfase na forma virchowiana - e no ano de 2016 houve um aumento de 47,05\% na notificação da forma tuberculóide no sexo feminino. Estabelecendo um comparativo com os dados coletados dos prontuários, observou-se uma congruência da forma clínica predominante - dimorfa -, seguida da forma tuberculóide, não classificada, indeterminada e virchowiana. 


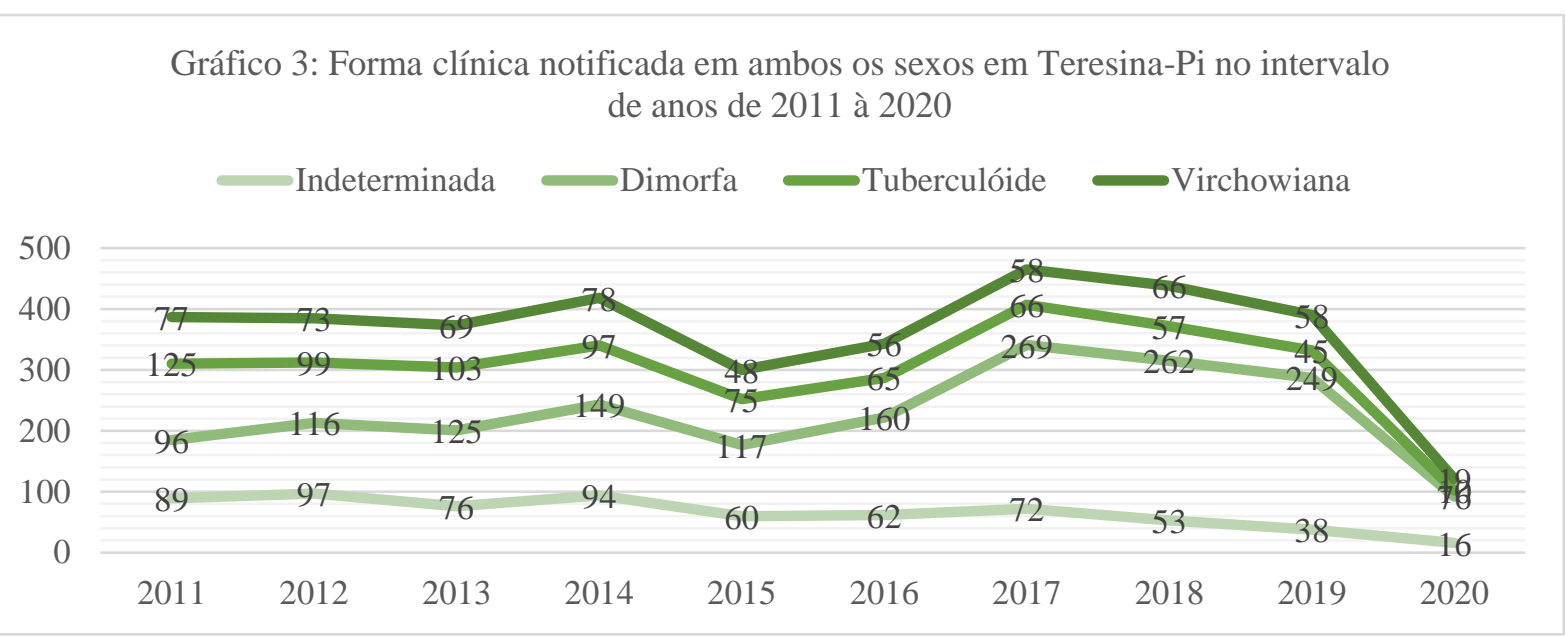

Fonte: Ministério da Saúde/SVS - Sistema de Informações de Agravos de Notificação - Sinan Net (2021).

Inerente à realização da baciloscopia (Gráfico 4), notou-se uma quantidade significativa da não realização de tal exame mediante diagnóstico da doença - o que reflete atualmente no diagnóstico não mais somente apresentação da positividade do exame baciloscópico, mas sim do conjunto de sinais e sintomas dermatoneurológicos que caracterizam a doença. Confirmando tais resultados, observou-se nos 46 prontuários avaliados que cerca de 54,35\% apresentou baciloscopia ignorada, 21,73\% apresentou a mesma positiva e $23,92 \%$ negativa - ressaltando que, de $54,35 \%$ dos pacientes que foram diagnosticados com a doença e tiveram a baciloscopia ignorada, $12,88 \%$ já tinham realizado o tratamento anteriormente e apresentavam contatos intradomiciliares que já realizaram tal tratamento.

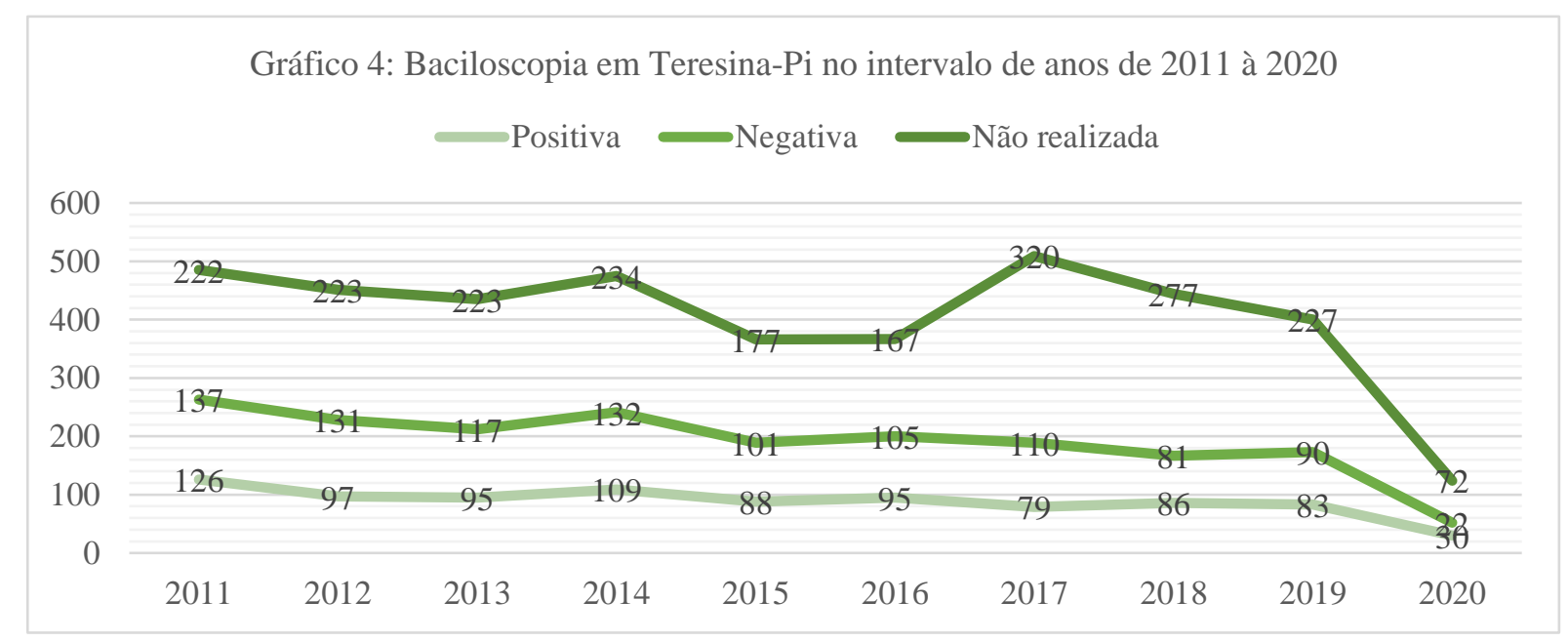

Fonte: Ministério da Saúde/SVS - Sistema de Informações de Agravos de Notificação - Sinan Net (2021).

Mediante o questionário para a avaliação de prontuários de pacientes submetidos ao tratamento para hanseníase adotado para a obtenção de tais dados, quanto as variáveis de uso de drogas lícitas - álcool e fumo, obteve-se os seguintes dados, expressos em percentagem (\%) na Tabela 1 : 
Tabela 1: Percentagem (\%) de usuários de drogas ilícitas entre os pacientes em tratamento para hanseníase nos anos de 2011 a 2021.

\begin{tabular}{|c|c|c|c|}
\hline Uso de drogas lícitas & Sim & Não & Não relata \\
\hline Álcool & $13,05 \%$ & $86,95 \%$ & $0 \%$ \\
\hline Fumo & $2,17 \%$ & $0 \%$ & $97,83 \%$ \\
\hline
\end{tabular}

Fonte: Autores (2021).

Em conjunto a tais variáveis não farmacológicas que auxiliam no tratamento da hanseníase, quanto a prática de exercício físico, somente 2 pacientes (4,34\%) relataram que realizavam frequentemente, enquanto 95,66\% relatam não realizar quaisquer exercícios físicos. Números semelhantes são observados quando aplicou-se a variável realização de fisioterapia como auxílio no tratamento da doença - onde somente 6,52\% (3 pacientes) relataram que já tinham realizado ou realizavam no momento do tratamento como auxílio para prevenção e recuperação dos nervos acometidos pelo bacilo de Hansen, enquanto 93,48\% relataram que não realizaram (43 pacientes), sendo que a maioria dos pacientes - no momento do preenchimento do prontuário - de (> 65\%) apresentou mais de um tronco nervoso comprometido e que necessitava da realização de sessões de fisioterapia como auxílio do tratamento para tal patologia.

Frente as variáveis farmacológicas, mediante o esquema terapêutico (Gráfico 5), notaram-se uma prevalência do esquema terapêutico de 12 meses, seguido pelo de 6 meses e pelo esquema terapêutico substituto - sendo que no ano de 2020 houve uma queda drástica em relação à ambos esquemas terapêuticos em comparação ao ano de 2019 (cerca de $70,46 \%$ em relação ao esquema de 12 meses, 66,21\% em relação ao esquema de 6 meses e 75\% em relação aos esquemas terapêuticos substitutos).

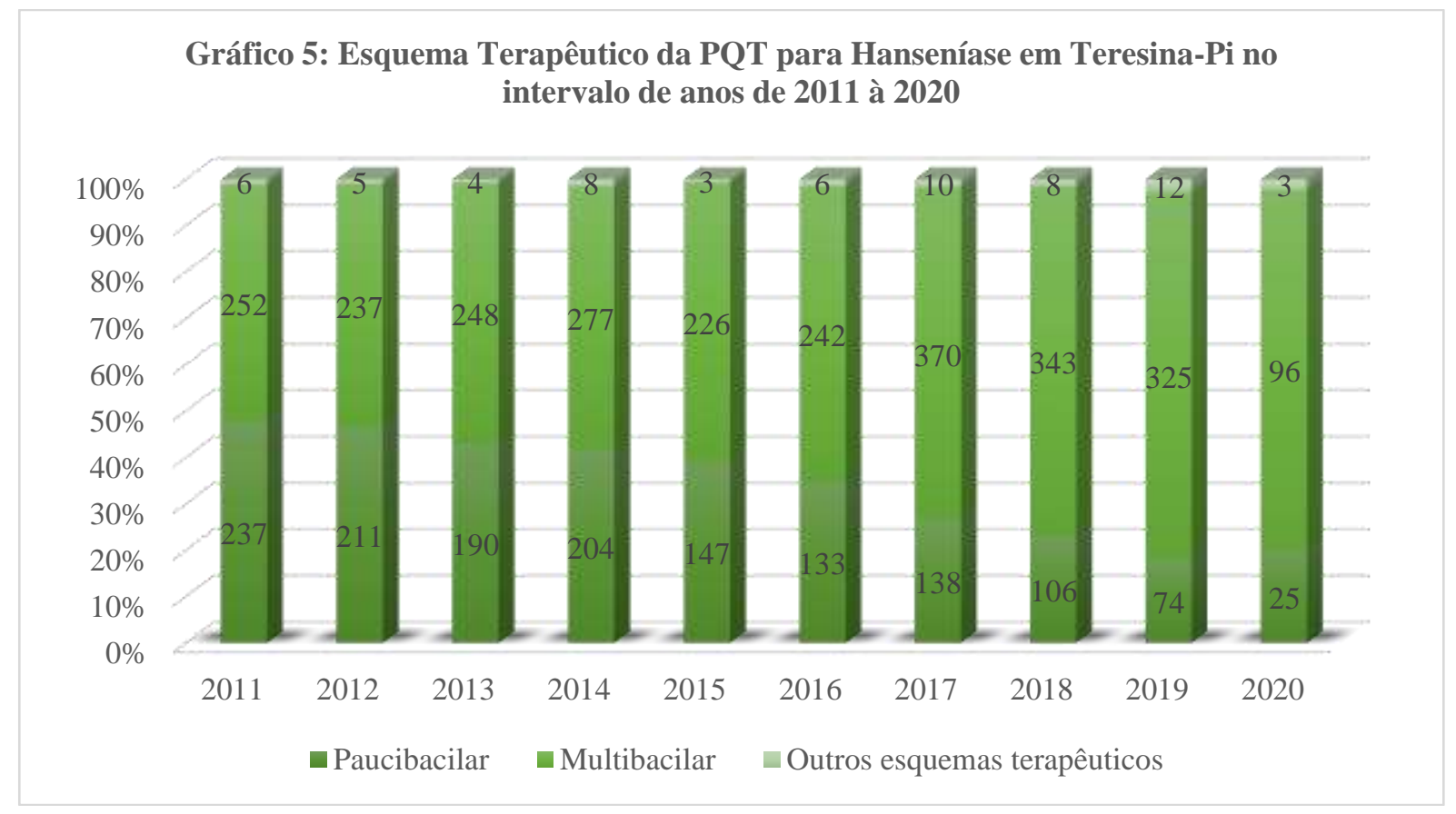

Fonte: Ministério da Saúde/SVS - Sistema de Informações de Agravos de Notificação - Sinan Net (2021).

Estabelecendo um comparativo entre os esquemas terapêuticos adotados e relatados nos prontuários dos pacientes, observou-se uma predominância semelhante ao observado nos dados apresentados no DATASUS - cerca de 63,04\% adotaram o esquema multibacilar, $24,48 \%$ o esquema paucibacilar, $6,52 \%$ adotaram outras drogas no tratamento para a doença e $4,34 \%$ 
alteraram o tratamento após seu início, sendo que tais pacientes tiveram alteração em sua terapia medicamentosa devido efeitos adversos associados à medicação.

Quanto à adesão à terapia farmacológica, avaliou-se a mesma através de duas variáveis: Se o paciente se esquece de tomar a medicação e seus horários de uso. Mediante tais perguntas estabelecidas, observou-se que, mediante a análise dos prontuários, cerca de $28,26 \%$ esqueciam-se as vezes de tomar a medicação, $13,04 \%$ sempre se esqueciam de tomar a medicação, 47,82\% nunca se esqueciam de tomar a medicação e 10,86\% não relatou. Quanto aos horários de uso da poliquimioterapia, 45,65\% relatou que usava no horário da tarde - cerca de duas horas após o almoço, como recomendado no início da terapia -, $52,17 \%$ não relatou e 2,18\% informou que utilizava em qualquer horário do dia.

Em conjunto à tais variáveis, avaliou-se também quanto à presença de comorbidades - onde cerca $65,22 \%$ informou apresentar alguma doença de caráter crônico diagnosticadas e, em relação à quantidade de patologias por paciente, 28,26\% relatou apresentar até uma doença, 19,56\% até duas, 10,86\% até três e 6,53\% apresentando mais de três doenças de caráter crônico diagnosticadas.

Dentre as diversas patologias de caráter crônico diagnosticadas, quanto a sua incidência em relação à quantidade de pacientes, observou-se cerca de 17 doenças enquadradas de caráter crônico, sendo que somente 4,34\% dos pacientes não souberam relatar que patologias apresentava - mas alegava que utilizava diversos medicamentos para o tratamento de alguma doença e que tinha o conhecimento que a patologia que apresentava era de caráter crônico. Assim, mediante os distúrbios apresentados, observou-se um predomínio de patologias hipertensivas (43,47\%), seguido das dislipidemias (19,56\%), diabetes $(17,39 \%)$ e anemia (13,04\%). Obesidade, osteoporose e patologias oculares - astigmatismo, hipermetropia e glaucoma - somadas totalizaram 13,04\% (apresentando somente dois casos cada), assim como hipertireoidismo, calculose renal, arritmia congênita, endometriose, dismenorreia, depressão, artrose, lúpus, esquizofrenia e epigastria somadas totalizaram $21,74 \%$ (apresentando somente um caso cada).

Decorrente da quantidade de patologias por paciente, avaliou-se concomitantemente a quantidade de fármacos utilizados pelos mesmos (Tabela 2) - com exceção das drogas administradas para tratamento da hanseníase -, assim como a quantidade de indivíduos que utilizam em associação outros medicamentos para tratamento das reações hansênicas e para os efeitos adversos desencadeados pelos fármacos da poliquimioterapia - onde a anemia e distúrbios gástricos foram os mais relatados (Tabela 3):

Tabela 2: Incidência de medicamentos utilizados por paciente para outras comorbidades entre os pacientes em tratamento para hanseníase nos anos de 2011 a 2021.

\begin{tabular}{|c|c|}
\hline Quantidade de medicamentos por pacientes & Total \\
\hline Até 1 medicamento & 6 \\
\hline Até 2 medicamentos & 7 \\
\hline Até 3 medicamentos & 9 \\
\hline Até 4 medicamentos & 4 \\
\hline Superior à 5 medicamentos & 8 \\
\hline
\end{tabular}

Fonte: Autores (2021).

Tabela 3: Incidência de medicamentos utilizados para o tratamento de reações - hansênicas e adversas - entre os pacientes em tratamento para hanseníase nos anos de 2011 a 2021.

\begin{tabular}{|c|c|c|}
\hline Tratamento & Fármacos & Total \\
\hline \multirow{2}{*}{ Reações hansênicas } & Talidomida associado à Prednisona & 1 \\
\cline { 2 - 3 } & Prednisona & 7 \\
\hline Anemia & Sulfato Ferroso 300mg/dia & 5 \\
\hline
\end{tabular}

Fonte: Autores (2021). 
Frente as interações medicamentosas avaliadas pelo software Medscape ${ }^{\circledR}$, as mesmas foram classificadas segundo a sua significância na terapia farmacológica em distúrbio maior (com necessidade de modificação da terapia por drogas alternativas devido elevado risco de vida ao paciente), distúrbio médio (com necessidade de monitoramento e possível substituição caso apresente riscos à vida do paciente) e distúrbio menor (com significância desconhecida e/ou reduzida).

Desta forma, de 46 prontuários avaliados, cerca de 32 pacientes relataram utilizar de outros medicamentos em conjunto à poliquimioterapia para tratamentos de patologias - sejam de caráter agudo como crônico. Desta amostra, ao ser avaliados mediante a possibilidade de interações medicamentosas através do software Medscape $®, 24$ pacientes apresentaram ao menos uma interação medicamentosa, onde 39,83\% desta amostra foi englobada em interações classificadas como distúrbio menor, $44,92 \%$ como distúrbio médio e $15,25 \%$ como distúrbio maior, onde as mesmas foram classificadas em outras 10 subclasses segundo a sua incidência em porcentagem, apresentados na tabela abaixo (Tabela 4):

Tabela 4: Classificação das interações medicamentosas segundo mecanismo de ação entre os pacientes em tratamento para hanseníase nos anos de 2011 a 2021.

\begin{tabular}{|c|c|c|}
\hline \multicolumn{2}{|c|}{ Classificação das interações medicamentosas segundo mecanismo de ação } \\
\hline Alterações no metabolismo hepático & Por indução enzimática & $33,90 \%$ \\
\hline Alterações na depuração renal & Por inibição enzimática & $6,77 \%$ \\
\hline Alterações na flora intestinal & $8,50 \%$ & $9,33 \%$ \\
\hline Alterações na glicoproteína P & $4,23 \%$ \\
\hline Sinergismo farmacodinâmico & $16,10 \%$ & $4,23 \%$ \\
\hline Antagonismo farmacodinâmico & $4,23 \%$ & $0,84 \%$ \\
\hline Alterações gastrointestinais & Inibição da absorção gastrointestinal \\
\hline Aumento da toxicidade no intervalo QTC & Estímulo da absorção gastrointestinal \\
\hline Alterações no pH gástrico & $4,23 \%$ & \\
\hline Deslocamento GABA & $3,40 \%$ & $0,84 \%$ \\
\hline Mecanismo desconhecido & $3,40 \%$ \\
\hline
\end{tabular}

Fonte: Autores (2021).

Visando um complemento das variáveis farmacológicas, avaliou-se em conjunto a incidência de reações adversas à poliquimioterapia, onde cerca de $50 \%$ dos pacientes relatou ao menos apresentar 2 efeitos adversos associados à medicação utilizada para tratamento da hanseníase. Assim, de acordo com a medicação estabelecida em cada esquema terapêutico, registrouse as reações adversas que se correlacionavam aos medicamentos utilizados, onde obteve-se os seguintes dados (Tabela 5): 
Tabela 5: Incidência de reações adversas segundo medicação da PQT entre os pacientes em tratamento para hanseníase nos anos de 2011 a 2021.

\begin{tabular}{|c|c|c|}
\hline \multirow{2}{*}{ Fármacos } & Reação adversa & $\begin{array}{c}\text { Percentagem em comparação à } \\
\text { população avaliada }\end{array}$ \\
\hline \multirow{3}{*}{ Rifampicina } & Diarreia & $4,35 \%$ \\
\cline { 2 - 3 } & Vômito & $2,18 \%$ \\
\cline { 2 - 3 } & Mal estar & $13,04 \%$ \\
\hline \multirow{2}{*}{ Dapsona } & Diminuição do apetite e náuseas & $17,40 \%$ \\
\hline \multirow{3}{*}{ Clofazimina } & Gastrite & $8,70 \%$ \\
\hline & Anemia & $8,70 \%$ \\
\cline { 2 - 3 } & Fotodermatite & $2,18 \%$ \\
\cline { 2 - 3 } & Hiperpigmentação cutânea & $17,40 \%$ \\
\cline { 2 - 3 } & Ictiose & $10,87 \%$ \\
\hline
\end{tabular}

Fonte: Autores (2021).

\section{Discussão}

De acordo com os resultados do estudo, a faixa etária predominante foi de 60-69 anos, dado também observado em uma pesquisa realizada com idosos em dois Centros de Reabilitação em São Luiz-Maranhão em 2017, dos quais 50,3\% tinham a mesma faixa de idade correspondente aos abordados em tal pesquisa.

Como tal faixa etária predominante, compreende-se assim que o intervalo de idade classifica a população como idosa. A parcela de idosos na população brasileira vêm crescendo gradativamente nos últimos anos, aumentando assim a expectativa de vida da população em decorrência de diversos avanços médicos, tecnológicos e ações de saúde pública - como vacinação e saneamento básico. Desta forma, observa-se o aumento da demanda de melhorias na atenção à saúde, sobretudo no tocante às deficiências da assistência farmacêutica prestada à população (Karnikowshi et.al., 2004).

O envelhecimento é um processo sequencial, individual, acumulativo, irreversível, universal, não patológico e próprio a todos os membros de uma espécie, onde têm-se a deterioração de um organismo maduro. Ao se associar às doenças crônicodegenerativas, observa-se um aumento na demanda aos serviços de saúde, o que corrobora para o uso de polifarmácia, reações adversas aos medicamentos (RAM's) e a efeitos iatrogênicos. Atualmente, os idosos são considerados o grupo populacional que mais cresce no Brasil e são os maiores consumidores de medicamentos no mundo.

Devido ao uso de medicamentos prescritos e não prescritos (automedicação), as reações adversas aos medicamentos tornaram-se uma das principais causas de admissão em serviços de saúde e um enorme problema de saúde pública. Contudo, deve-se atentar as mudanças nas condições fisiológicas decorrentes da idade que não devem ser desconsideradas, pois podem acarretar em uma farmacocinética diferenciada e, desta forma, maior sensibilidade tanto aos efeitos terapêuticos como aos adversos dos fármacos.

Com relação à variável sexo, têm-se conhecimento que a hanseníase atinge homens e mulheres, mas alguns trabalhos apontam para prevalência da enfermidade no sexo masculino, como relata a Organização Mundial da Saúde (OMS) em seu estudo Estratégia Global para Hanseníase 2016-2020: Aceleração rumo a um mundo sem hanseníase, que somente 36\% dos novos casos em 2014 eram mulheres. No Brasil, um estudo epidemiológico realizado no município de Araçatuba-São Paulo cidade considerada endêmico no Brasil para tal patologia -, examinou-se cerca de 434 casos durante o ano de 2015 e encontrou uma distribuição maior em mulheres - cerca de 52,5\% dos casos - contudo com uma diferença de 5\% entre o sexo masculino, que foi representado por $47,5 \%$ dos casos. 
Mediante tal contexto, torna-se evidente o reflexo dos anos de negligência com a saúde do homem, tornando-os mais vulneráveis a patologias de caráter grave e crônico decorrente da ausência de hábitos de prevenção de doenças. A partir de tal concepção, o Ministério da Saúde implementou em 2009 a Política Nacional de Atenção Integral à Saúde do Homem que visa o oferecimento de ações voltadas à tal público associado à promoção da saúde.

Embora a hanseníase possa atingir ambos os sexos, o descuido frente a procura aos serviços de saúde, a menor preocupação com o processo saúde-adoecimento associado à uma patologia característica crônica e a transmissão da doença decorrente de contato prolongado sem tratamento pode cooperar com a prevalência de tal enfermidade nesse sexo, contribuindo consequente para o maior risco de adoecimento.

Associando ao grau de escolaridade, tal estudo não contradiz à realidade atual em comparação à outras doenças, já que a maior ocorrência da patologia concentrou-se em indivíduos que não concluíram todas as etapas de ensino - correspondendo à soma de pacientes que relataram não terem concluído o ensino primário, fundamental, médio e superior. Desta forma, enquanto problema de saúde pública, a hanseníase está fortemente atrelada ao contexto social que acaba por refletir nos determinantes e condicionantes de saúde aos quais o indivíduo é submetido, contribuindo significantemente para o aumento do risco de adoecimento e colaborando com o estigma e negligência à doença - onde a mesma encontra-se atrelada as condições socioeconômicas, corroborando com a sua resolução enquanto problema de saúde pública.

A adesão correta à terapia farmacológica vem sendo estudada e discutida em diversas literaturas. Sua importância é crucial para o tratamento de um paciente e para a melhora do quadro de saúde do mesmo. Assim, evidenciou-se que cerca de $28,26 \%$ e $13,04 \%$ da população em análise relatou que esqueciam-se "às vezes" e "sempre", respectivamente, de utilizar os medicamentos para o tratamento da hanseníase, sendo que seu uso de forma contínua durante a poliquimioterapia é de caráter crucial para uma correta resolubilidade da doença. Contudo, o processo de adesão engloba, geralmente, uma diversidade de fatores. Entre os mesmos, pressupostos pessoais do paciente interferem diretamente na adesão - somado ao tempo de uso, cultura, número de medicamentos utilizados, associação de fármacos em um único medicamento, etc. -, o que acaba por tornar fundamental uma avaliação particular de cada caso para que haja uma adequada compreensão de fatores que possam promover ou não a adesão à terapia. O conhecimento acerca da doença tratada é imprescindível pois, com o desaparecimento dos sintomas responsáveis pela queixa inicial, o paciente tende a descontinuar o tratamento, o que acarreta em uma inadequada adesão e resolubilidade terapêutica.

Avaliando especificamente cada caso, o conhecimento dos fatores que levam ao uso ou não de fármacos prescritos por um profissional permite a criação de melhores maneiras de demonstrar ao paciente a real importância de determinado tratamento na mudança da sua condição atual (Mourão Jr \& Souza, 2010). Adversidades oriundas do uso incorreto ou até do não uso dos medicamentos para o tratamento de certa condição clínica podem ser evitados, ressaltando assim uma importância de caráter crucial do profissional prescritor e da equipe multidisciplinar que acompanham o paciente acerca da necessidade do uso correto da medicação. Ao ouvir e entender adequadamente o paciente, o mesmo também será compreendido, fornecendo posteriormente orientações visando uma adequada adesão ao tratamento.

A hanseníase pode ser classificada de acordo com o número de lesões dermatológicas - até cinco lesões e superior à cinco lesões -, agrupando assim a hanseníase em paucibacilar (PB) - que engloba as formas clínicas indeterminada e tuberculóide - e multibacilar (MB), com as formas dimorfa e virchowiana. O estudo apresenta uma predominância na forma clínica dimorfa e, consequentemente, dos casos multibaciliares, com mais de cinco lesões dermatológicas. Em contrapartida, chama-se atenção aos casos ignorados e negativos de baciloscopia - o que reforça o diagnóstico não mais somente mediante positividade do exame baciloscópico, mas sim do conjunto de sinais e sintomas dermatoneurológicos que caracterizam a doença. Objetivando estabelecer um comparativo, observou-se outros trabalhos de caráter epidemiológico que correlacionam tais variáveis e que apresentaram congruência com os obtidos: um estudo realizado em Fortaleza-Ceará no período de 2007-2011 e no estado de 
Alagoas no período de 2005-2015. Dados similares com estudo realizado no continente Africano foram obtidos onde, em média, observou-se um predomínio da forma dimorfa e classificação operacional MB, somado ao quantitativo superior de cinco lesões tegumentares.

A incapacidade física decorrente das deformidades devido o processo inflamatório ocasionado pela doença é considerada um dos principais problemas da hanseníase pois desencadeia um comprometimento físico e psicológico, associado ao sentimento de exclusão, estigma e preconceito que afetam diretamente a pessoa. $\mathrm{O}$ acometimento neural surge devido a predileção do bacilo de Hansen pelos nervos periféricos - o que torna assim imprescindível a realização de uma avaliação neurofuncional tanto no momento do diagnóstico como durante o tratamento e na alta visando a investigação de possíveis alterações motoras e neurológicas, auxiliando assim no tratamento das mesmas com agilidade e redução de agravos através da realização de sessões de fisioterapia e prática de exercícios físicos como medidas preventivas.

Desta forma, ao serem avaliados as variáveis não farmacológicas do tratamento, notou-se que somente 4,34\% relatou a prática de exercício físico e $6,52 \%$ que realizavam sessões de fisioterapia como auxílio do tratamento da doença - sendo que $62,35 \%$ apresentou, no momento do diagnóstico, algum grau de incapacidade ocasionado pela doença, seguido pelas respostas de "não avaliado" e "ignorado", evidenciando assim uma negligência pelos profissionais de saúde para com os pacientes pois a avaliação colabora para a adoção de práticas preventivas e no planejamento para promoção e continuidade dos cuidados em saúde. Tal fato pode ser considerado alarmante pois grande parte dos prontuários avaliados relatavam a incidência de casos multibacilares classificados como dimorfa, predominante a faixa de idade idosa - o que favorece um possível comprometimento neural em pacientes que já possuem um risco para déficit funcional.

Quanto ao uso de drogas lícitas - álcool e fumo - mesmo apresentando baixa incidência de sua utilização pelos pacientes $(13,05 \%$ e 2,17\%), salienta-se que o etanol desencadeia alterações de caráter farmacocinético e farmacodinâmico notórias quando usado concomitantemente com fármacos, pois o mesmo acelera o esvaziamento gástrico - alterando assim biotransformação de fármacos - e interfere em sítio de ação dos fármacos - por meio de modificação em receptores, neurotransmissores ou efetores, reforçando ou inibindo efeitos de outros fármacos, atuando em sítios diferentes e por mecanismos diversos.

Acerca do fumo, têm-se conhecimento dos diversos danos em variados órgãos e sistemas ocasionado pelas mais de 4.000 substâncias que compõem a fumaça - algumas das quais apresentam ação farmacológica ativa, citotóxica, antigênica, mutagênica, incluindo pelo menos 43 carcinógenos -, sendo assim geradora de um largo espectro de efeitos deletérios ao organismo, onde o coração, aparelho gastrointestinal, pulmões, sangue e sistemas imune e nervoso são os mais afetados. Classificado como um alcaloide, a nicotina exerce ação estimulante e depressora ganglionar dose-dependente, demonstrado em diversos estudos também a sua capacidade de alteração na farmacocinética de diversas drogas - sendo o responsável pela indução do metabolismo por enzimas, modificando assim a meia-vida de drogas processadas pelo fígado e diminuição na concentração plasmática da droga e consequentemente seus efeitos farmacológicos.

Sendo um problema também identificado em outras pesquisas, o registro incompleto de dados acaba por afetar diretamente as possíveis estratégias a serem adotadas para o aumento da vigilância epidemiológica - como o aperfeiçoamento técnico das equipes de saúde atuantes nos serviços de saúde nas quais a ausência de dados dos casos ou suas subnotificações são mais evidentes visando investigar e acompanhar cada caso - e também a evidência da necessidade de uma equipe multiprofissional que acompanhe diretamente cada paciente e suas peculiaridades fisiopatológicas atreladas à sua condição de saúde.

Mediante a avaliação da quantidade de fármacos utilizados por pacientes - com exceção dos medicamentos administrados para tratamento da hanseníase - assim como a quantidade de indivíduos que utilizam em associação outros medicamentos para tratamento das reações hansênicas e para os efeitos adversos desencadeados pelos fármacos da poliquimioterapia, evidenciou-se o uso de polifarmácia - sendo este termo utilizado para definir, segundo estudos norte- 
americanos, como "a prescrição, administração ou uso de mais medicamentos do que está clinicamente indicado". Em contraste, estudos latino-americanos e europeus utilizaram o conceito quantitativo que engloba "o uso simultâneo de vários medicamentos".

Com a prevalência de doenças crônicas não transmissíveis devido ao aumento da taxa de envelhecimento populacional durante os últimos dez anos - com influência direta da elevação da expectativa de vida e do crescimento de tal faixa de idade -, têm-se uma predisposição do idoso à polifarmácia, respectivamente às reações adversas aos medicamentos e à prescrição dos mesmos realizados de forma inadequada. Pesquisas apontam que o uso de polifarmácia pode contribuir para o uso incorreto dos seus fármacos e possíveis complicações das doenças causadas pela ineficácia do tratamento ou por eventos adversos, levando ao aumento da morbimortalidade e dos custos dos tratamentos. Infelizmente, é escassa a implementação de tratamentos não farmacológicos no Brasil, em contrapartida ao acesso aos medicamentos que é de elevada facilidade, o que acaba por contribuir para a automedicação e inúmeras consequências à saúde de seus usuários.

Com a avaliação da polifarmácia, observou-se que cerca de 69,56\% dos pacientes relatou o uso de mais de um medicamento associados à poliquimioterapia e, desta população, 54,34\% apresentou, ao ser avaliado - entre os medicamentos utilizados concomitantemente com os fármacos para tratamento da hanseníase -, interações entre as drogas utilizadas, onde as alterações à nível de metabolismo hepático através da indução enzimática é a que apresentara índices mais elevados, seguidos pelo sinergismo farmacodinâmico, alteração na flora intestinal, alteração na depuração renal e inibição enzimática.

A indução enzimática é um processo farmacocinético onde ocorre a estimulação da atividade das enzimas microssomais hepáticas - no caso, as que são compreendidas na família do citocromo P450, subfamília CYP3A4, CYP2C19 e CYP2C9 - por medicamentos ou outras substâncias e é considerado um notório problema clínico pois a estimulação da biotransformação (seja própria ou desencadeado por outra substância) acarreta no aumento da velocidade da produção de metabólitos e na depuração hepática da droga, somado à redução da meia-vida sérica do fármaco - o que afeta diretamente as suas concentrações livre e total e efeito farmacológico. A rifampicina é considerado o principal indutor enzimático dentre as interações medicamentosas analisadas - reduzindo os efeitos da clofazimina e dapsona (fármacos compreendidos nos esquemas paucibacilar e multibacilar da hanseníase), seguido da prednisona - antiinflamatórios esteroidal utilizado no tratamento das reações hansênicas tipo I e II. Ressalta-se que a rifampicina também atua à nível de glicoproteína $\mathrm{P}$, especificamente reduzindo o influxo do fármaco para o interior da célula, e consequentemente do efeito terapêutico.

Evidenciou-se que a ocorrência de reações adversas é decorrente do tratamento poliquimioterápico, que em alguns casos pode impedir o paciente de atividades do cotidiano ou até mesmo torná-lo incapaz. Com isso a importância das consultas mensais para a avaliação do tratamento e estados reacionais da doença. De acordo com os prontuários analisados a rifampicina foi apontada como principal responsável pela maioria das reações relatadas pelos indivíduos, causando diarreia, náuseas, vômitos, mal-estar e a diminuição do apetite. A dapsona destaca-se como efeitos adversos casos de anemia, gastrite e foto dermatite. Assim como a clofazimina provoca alterações cutâneas - pele seca, hiperpigmentação cutânea, ictose e pigmentação escura na pele -, portanto é indispensável as informações aos pacientes que durante o período de tratamento evitar exposição a luz solar, ingerir bastante água e manter a pele hidratada.

Os pacientes devem ser informados acerca dos efeitos adversos mais comuns e devem ser orientados a relatar ao profissional da saúde para melhor conduta. Devido ao tratamento ser considerando longo e com a existência de tais efeitos, têmse o abandono do uso dos medicamentos, sendo justificado pela ausência durante o acompanhamento mensal para a dose supervisionada dos fármacos. A dapsona é metabolizada no fígado, sendo responsável pela produção de hidroxilamina, e pelas alterações hematológicas do medicamento, como a metahemoglobina e anemia hemolítica (Carvalho C, et al., 2011). Evidenciase assim importância de um acompanhamento por exames laboratoriais frequentemente e registro nos prontuários os resultados, pois alguns dos pacientes avaliados necessitaram do uso de uma suplementação para deficiência de ferro. Com a clofazimina, os efeitos - alterações cutâneas - estão associados a dose, que com a suspensão do medicamento podem ser reversíveis. A 
rifampicina é a causadora das alterações gastrointestinais, quando identificada é sugerido medicamentos para alivio de tais reações, como os inibidores da bomba de prótons.

A inibição da absorção gastrointestinal é outra interação de grande importância a ser discutida pois, através de tal mecanismo farmacocinético, têm-se consequentemente a redução dos níveis da droga e seus efeitos, sendo evidenciado tal interação entre a clofazimina e a rifampicina. Também atuando à nível gastrointestinal, têm-se a dapsona - que ocasiona um desequilíbrio na flora intestinal, pois além de desencadear uma lise no Mycobacterium leprae, propicia a morte da microbiota intestinal presente no intestino que auxiliam na absorção de substâncias, como diversas vitaminas utilizadas no tratamento da hanseníase (piridoxina, tiamina e ácido pantotênico).

A prednisona é o principal fármaco utilizado no tratamento da hanseníase visando intervir nas reações hansênicas tipo I e II - sendo que nas reações tipo II têm-se também o uso da talidomida em casos mais graves. Possui elevada eficácia antiinflamatória que auxilia na redução das dores de caráter neuropático ocasionadas pela doença. Contudo, apresenta diversos efeitos colaterais que são proporcionais ao tempo e dose de uso, como o aumento dos níveis glicêmicos no sangue - o que faz jus ao outro nome dos fármacos corticosteroides: glicocorticoides.

Através do estímulo da secreção de glicose pelo fígado, assim como pela redução do transporte de glicose em células adiposas e musculares, a prednisona induz níveis elevados de glicose, desencadeando um "efeito global" na redução da depuração de glicose. Níveis elevados de glicose podem acarretar em sua toxicidade, prejudicando a secreção de insulina, assim como a expressão do GLUT-2 - que é um transportador de glicose mediado por proteína que transporta glicose através das membranas celulares. Desta forma, pacientes diabéticos em uso de prednisona terão um aumento significativo nos níveis séricos de glicose, que é diretamente proporcional à dose do esteroide administrada.

Notou-se assim que, de 46 pacientes submetidos ao tratamento para hanseníase, 17,4\% possui diabetes e 13,04\% utilizou prednisona em algum momento do tratamento visando a redução da incidência das reações hansênicas. Desta forma, a curva de ação da glicose - que geralmente se elevam após o almoço e permanecem até o início da noite - dificulta a ação dos hipoglicemiantes - pois a prednisona administrada pela manhã não causa elevados níveis de glicose momentaneamente - e, consequentemente, reduz a eficácia da terapia medicamentosa adotada no tratamento da diabetes. A negligência à tal efeito adverso ocasionado pelo glicocorticoide está intrinsicamente relacionado à piora do quadro diabético do paciente oriunda dos efeitos deletérios ocasionados pelo aumento prolongado da glicose sanguínea.

Com seu uso sendo recomendado no tratamento da hanseníase visando a redução do desconforto estomacal ocasionado pelos fármacos que compõem a poliquimioterapia, o Omeprazol é um medicamento pertencente à classe dos inibidores da bomba de prótons (PPIs), atuando através da supressão da secreção de ácido gástrico pelas células parietais do estômago especificamente, pela inibição da enzima $\mathrm{H}^{+} \mathrm{K}^{+} \mathrm{ATPase}$ - que pode durar até 48 horas. Por apresentarem poucos efeitos adversos quando administrados corretamente, tal medicamento passou a não ser usado somente para sintomas agudos na prática clínica, mas para qualquer distúrbio gástrico.

Evidenciou-se assim que $24 \%$ dos pacientes que apresentou pelo menos uma interação medicamentosa utilizou tal fármaco para o tratamento de algum distúrbio gástrico ocasionado pela PQT em um período superior há 8 semanas, que é o recomendado em casos de esofagite de refluxo. Constatou-se em estudos epidemiológicos que há uma relação entre o uso prolongado de PPIs e o metabolismo ósseo: Yang et al. descreveram que a administração de Omeprazol (20mg/dia) é capaz de reduzir significantemente a densidade mineral óssea - onde o mecanismo de elevação do $\mathrm{pH}$ gástrico está como o principal causador, pois interfere na absorção do cálcio (que é um sal insolúvel em pH básico e, desta forma, menos absorvido). 


\section{Considerações Finais}

Desta forma, mediante os resultados apresentados e discutidos, conclui-se que a adesão à terapia farmacológica e não farmacológica da terapêutica dos pacientes submetidos ao tratamento para hanseníase entre 2011 à 2021 encontra-se comprometida mediante, em destaque, do desconhecimento dos pacientes acerca da doença - em conjunto dos diversos efeitos adversos desencadeados pela poliquimioterapia e o inexistente acompanhamento farmacoterapêutico dos mesmos, com a ausência da elucidação das possíveis reações adversas aos medicamentos (RAM's) da terapia anti-hansênica.

Destaca-se também que pesquisas utilizando bases de dados de domínio público podem minimizar custos e tempo, constituindo-se em fonte segura para pesquisas e organização de serviços e políticas públicas. Assim, por meio do presente estudo, ficou evidente que os recursos utilizados forneceram um panorama epidemiológico dos casos de hanseníase diagnosticados no Brasil em indivíduos com idade de 20 a 75 anos, podendo ser uma ferramenta útil para os profissionais de saúde repensarem a sua prática e direcionarem investimentos nesta área do conhecimento, além de oportunizarem aos gestores públicos o planejamento de estratégias preventivas específicas a esta população. Ressalta-se também que o conhecimento epidemiológico nesta população é essencial para o direcionamento das ações de promoção, prevenção e reabilitação.

Por fim, verificou-se a necessidade da implementação do profissional farmacêutico na equipe de cuidado do paciente com hanseníase, sendo de fato o responsável pela farmacoterapia do indivíduo, inserindo-o na equipe multiprofissional do cuidado, assumindo a responsabilidade pelo cuidado do mesmo - contribuindo desde a identificação dos sinais e sintomas da doença e o encaminhamento do paciente a uma UBS ou unidade de referência para melhor investigação. O farmacêutico como profissional do medicamento é essencial no auxílio da escolha dos fármacos e na orientação do seu uso correto na PQT, a fim de minimizar os seus possíveis efeitos colaterais, reforçando a importância da conclusão do tratamento para obtenção da cura da doença e sucesso na farmacoterapia do paciente, reafirmando o seu papel na equipe de saúde e demonstrando o seu valor social.

\section{Agradecimentos}

Agradecemos a todos que participaram, direta ou indiretamente do desenvolvimento deste trabalho de pesquisa, enriquecendo o meu processo de aprendizado. Em especial, a Deus, pela vida e por nos permitir ultrapassar todos os obstáculos encontrados ao longo da realização deste trabalho. Aos colaboradores da Unidade Básica de Saúde São Camilo, pelo acolhimento, atenção e auxílio durante todo o processo de coleta de dados. À nossa professora orientadora Alessandra Camillo, por ter sido nossa orientadora e ter desempenhado tal função com dedicação e amizade. Aos amigos e familiares, por todo o apoio e pela ajuda, que muitos contribuíram para a realização deste trabalho.

\section{Referências}

Araújo, M. G. (2003). Hanseníase no Brasil. Rev. Soc. Bras. Med. Trop. 36(3), 373-82.

Barbosa, D. R. M., Almeida, M. G., \& Santos, A. G. (2014). Características epidemiológicas e espaciais da Hanseníase no Estado espaciais da Hanseníase no Estado do Maranhão, Brasil, 2001-2012. Medicina (Ribeirão Preto). 47(4), 347-56.

Bechler, R. G. (2012). Hansen versus Neisser: controvérsias científicas na 'descoberta' do bacilo da lepra. Hist. cienc. Saúde - Manguinhos, Rio de Janeiro. 19(3), $815-42$.

Borba, S. M. L. S. (2015). Vigilância epidemiológica da hanseníase na atenção básica: o caso do município de Itaboraí, região metropolitana do Rio de Janeiro. Escola Nacional de Saúde Pública Sergio Arouca, Rio de Janeiro -- 2015. xv,164 p.

Brasil (2007). Conselho Nacional de Secretários de Saúde. Assistência Farmacêutica no SUS / Conselho Nacional de Secretários de Saúde. - Brasília: CONASS, $186 \mathrm{p}$.

Brasil (2016). Ministério da Saúde. Gabinete do Ministro. Portaria nº 149, de 3 de fevereiro de 2016. Aprova as Diretrizes para Vigilância, Atenção e Eliminação da Hanseníase como Problema de Saúde Pública, com a finalidade de orientar os gestores e os profissionais dos serviços de saúde. Diário Oficial da União, Brasília, DF, n. 24, 4 fev. Seção 1, p. 45.

Brasil (2000). Ministério da Saúde. Portaria No 1073/GM de 26 de setembro de 2000. Publicada no D.O.U. - 188 -E -pg 18 -Seção 1 - 28 de setembro. 
Brasil (2008). Ministério da Saúde. Secretaria de Atenção à Saúde. Departamento de Atenção Básica. Vigilância em Saúde: dengue, esquistossomose, hanseníase, malária, tracoma e tuberculose. 2. ed. rev., Brasília. (Cadernos de Atenção Básica, n. 21).

Brasil (2010). Ministério da Saúde. Secretaria de Vigilância em Saúde. Departamento de Vigilância Epidemiológica. Capacitação em prevenção de incapacidades em hanseníase: caderno do monitor. Brasília.

Brasil (2001). Ministério da Saúde. Secretaria de Políticas de Saúde. Departamento de Atenção Básica. Controle da hanseníase na atenção básica: guia prático para profissionais da equipe de saúde da família / Ministério da Saúde, Secretaria de Políticas de Saúde, Departamento de Atenção Básica; elaboração de Maria Bernadete Moreira e Milton Menezes da Costa Neto. - Brasília: Ministério da Saúde.

Brasil (2002). Ministério da Saúde. Secretaria de Políticas de Saúde. Departamento de Atenção Básica. Guia para o Controle da hanseníase. Brasília: Ministério da Saúde.

Brasil (2017). Ministério da Saúde. Secretaria de Vigilância em Saúde. Departamento de Vigilância das Doenças Transmissíveis. Guia prático sobre a hanseníase [recurso eletrônico] / Ministério da Saúde, Secretaria de Vigilância em Saúde, Departamento de Vigilância das Doenças Transmissíveis. - Brasília: Ministério da Saúde, 68p.

Brasil (2019). Ministério da Saúde. Secretaria de Vigilância em Saúde. Departamento de Vigilância de Doenças e Agravos não Transmissíveis e Promoção da Saúde. Saúde Brasil 2018. Uma análise de situação de saúde e das doenças e agravos crônicos: desafios e perspectivas / Ministério da Saúde, Secretaria de Vigilância em Saúde, Departamento de Vigilância de Doenças e Agravos Não Transmissíveis e Promoção da Saúde - Brasília: Ministério da Saúde, 424 p.

Chakora, E. S (2014). Política Nacional de Atenção Integral à Saúde do Homem. Esc Anna Nery [Internet];18(4):559-61.

Diniz, L. M., Catabriga, M. D. S., \& Souza Filho, J. B (2010). Avaliação de hansenianos tratados com esquema alternativo dose única ROM (rifampicina, ofloxacina e minociclina), após sete a nove anos [Evaluation years in leprosy patients treated with single dose alternative scheme ROM (rifampin, ofl oxacin, minocycline), after seven to nine]. Rev Soc Bras Med Trop.;43(6):695-9.

Eizerik, D. P., Costa, A. F., \& Manfroi, W. C. (2008). Educação de pacientes em dislipidemia; Revista Brasileira de Farmácia; 89(3): 207-210.

Foss, N. T (1999). Hanseníase: aspectos clínicos, imunológicos e terapêuticos. Anais Brasileiros de Dermatolologia. 74:113-119.

Goulart, I. M. B., Penna, G. O., \& Cunha, G. (2002). Imunopatologia da hanseníase: a complexidade dos mecanismos da resposta imune do hospedeiro ao Mycobacterium leprae. Rev. Soc. Bras. Med. Trop., Uberaba, v. 35, n. 4, p. 363-375.

Jardim, M. R., Antunes, S. L., Santos, A. R., Nascimento, O. J, Nery, J. A., Sales, A. M. et al (2003). Criteria for diagnosis of pure neural leprosy. J Neurol; 250:806-9.

Latória, J. C., \& Abreu, M. A. M. (2012). Hanseníase: diagnóstico e tratamento. Diagnóstico \& Tratamento, 17, $173-179$.

Laurenti, R., Jorge, M. H. P. M., \& Gotlieb, S. L. D. (2005). Perfil epidemiológico da morbi-mortalidade masculina. Ciênc. saúde coletiva, $10,35-46$.

Legendre, D. P., Muzny, C. A., \& Swiatlo, E. (2012). Hansen's disease (Leprosy): current and future pharmacotherapy and treatment of disease-related immunologic reactions. Pharmacotherapy. Jan; 32(1):27-37.

Lima, L.S. (1953). Estado atual da terapêutica da lepra. Ministério da Educação e Saúde, São Paulo.

Luna, I. T. et al. (2010). Adesão ao tratamento da Hanseníase: dificuldades inerentes aos portadores. Rev. bras. enferm., 63, 983-990.

Madureira, A. M. A. S. (2015). Doenças Emergentes e Re-Emergentes na Saúde Coletiva. 1.ed. Instituto Federal do Norte de Minas Gerais.

Marciano, L. H. S. C., Belone, A. F. F., Rosa, P. S., Coelho, N. M. B., Ghidella, C. C., Nardi, S. M. T., Miranda, W. C., Barrozo, L. V., \& Lastória, J. C. (2018) Epidemiological and geographical characterization of leprosy in a Brazilian hyperendemic municipality. Cad Saude Publica, 20;34(8).

Martins, R. J., Carloni, M. E. O. G., Moimaz, S. A. S., Garbin, C. A. S., \& Garbin, A. J. I. (2016). Sociodemographic and epidemiological profile of leprosy patients in na endemic region in Brazil. Rev Soc Bras Med Trop [Internet]; 49(6):777- 80.

Mendonca, V. A. et al. (2008). Imunologia da hanseníase. An. Bras. Dermatol. 83, 343-350.

Moreira, A. S. et al. (2006) Baciloscopia da conjuntiva no diagnóstico e acompanhamento de pacientes portadores de hanseníase. Arq. Bras. Oftalmol., 69, -869.

Mutarelli, E. G., Coelho, F. F., \& Haddad, M. S. (2000). Distúrbios de sensibilidade - Propedêutica neurológica: do sintoma ao diagnóstico. Sarvier; 59-78.

Negesse. Y., Beimnet, K., Miko, T., Wondimus, A., \& Berhan, T. Y. (1993). In leprosy the presence of mycobacteria in the nerve is an essential factor in the cycle and spectrum of Mycobacterium leprae infection. Lepr Rev. 64(2):104-9.

Opromolla, D. V. A. (1997). Terapêutica da Hanseníase. Medicina, 30, 345-350.

Pereira, L. R. L., \& Freitas, O. (2008). A evolução da Atenção Farmacêutica e a perspectiva para o Brasil. Rev. Bras. Cienc. Farm., 44, 601-612.

Pinheiro, M. G. C., Miranda, F. A. N., Simpson, C. A., Carvalho F. P. B., Ataide, C. A. V., \& Lira A. L. B. C. (2018). Understanding "patient discharge in leprosy": a concept analysis. Rev Gaucha Enferm. 38(4): e 63290.

Pinho, J. R. R., Andrade Junior H. F., \& Schenber, A. C. (1998). Os diferentes testes cutâneos existentes para acompanhamento de pacientes com hanseníase [Different cutaneous tests for follow-up of leprosy patients]. Hansen Int.;23(1/2):49-52. 
Research, Society and Development, v. 10, n. 12, e305101219698, 2021

(CC BY 4.0) | ISSN 2525-3409 | DOI: http://dx.doi.org/10.33448/rsd-v10i12.19698

Savassi, L. C. M. (2010). Hanseníase: políticas públicas e qualidade de vida de pacientes e seus cuidadores. Dissertação (Mestrado) - Dissertação para obtenção do título de Mestre em Ciências pelo Programa de Pós-Graduação em Ciências da Saúde do Centro de Pesquisas René Rachou. Área de concentração: Saúde Coletiva. Belo Horizonte, 179 p.

Silva, V. L., Aguiar, M. I. F., Vasconcelos, P. F., \& Aquino, D. M. C. (2017). Aspecto físico e as repercussões na qualidade de vida e autonomia de idosos afetados por Hanseníase. Enferm Glob 46:350- 61.

Silveira, M. G. B., Coelho, A. R., Rodrigues, S. M., Soares, M. M., \& Camillo, G. N. (2014). Portador de hanseníse: impacto psicológico do diagnóstico. Psicol Soc 26(2):517-27.

Organização Mundial da Saúde (2016). Estratégia mundial de eliminação da lepra 2016-2020: acelerar a ação para um mundo sem lepra

Ramos, A. C. V., Yamamura, M., Arroyo, L. H., Popolin, M. P., Chiaravalloti Neto, F., Palha, P. F. et al. (2017). Spatial clustering and local risk of leprosy in São Paulo, Brazil. PLoS Negl Trop Dis 11(2):1-15.

Yang, Y. X., Lewis, J. D., Epstein, S., \& Metz, D. C. (2006). Long-term próton pump inhibitor therapy and risk of hip fracture. JAMA; 296(24):2947-53 